\title{
Downstream and soaring interfaces and vortices in 2-D stratified wakes and their impact on transport of contaminants
}

\author{
Y. D. Chashechkin, V. V. Mitkin, and R. N. Bardakov \\ Laboratory of Fluid Mechanics, Institute for Problems in Mechanics of the RAS, 101/1, prospect Vernadskogo, Moscow, \\ 119526, Russia
}

Received: 11 October 2005 - Revised: 27 March 2006 - Accepted: 27 March 2006 - Published: 10 August 2006

\begin{abstract}
The flow of continuously stratified fluids past obstacles was studied analytically, numerically, and experimentally. The obstacles discussed here include a flat strip, aligned with the flow, inclined or transverse to the flow and a horizontal cylinder. In the flow pattern, transient and attached (lee) internal waves, downstream wakes with submerged interfaces and vortices, soaring singular interfaces, soaring vortices and vortex systems are distinguished. New components of laminar flow past a horizontally towed strip are presented. Fine transverse streaky structures on the strip in the downstream wake were visualized. Soaring isolated interfaces, which are internal boundary layers forming inside the downstream attached wave field past bluff bodies were observed. With increasing of the body velocity a vortex pair was formed directly at the leading edge of this interface.
\end{abstract}

\section{Introduction}

Interest in the study of fine structure effects on mixing in a stratified flow is stimulated by a number of environmental and technological problems. Even when the density changes are small, density gradients can be large and lead to some new phenomena, which are not present in a homogeneous or continuously stratified environment. Links between internal waves and so-called "fine structure", which manifests itself by high gradient thin interfaces separating thicker and more homogeneous layers are under investigation.

It is well-known that stratification strongly affects flow separation and downstream wake structure. Such flow configurations are encountered in the atmosphere in the lee of hills or mountains and behind long ridges in the oceans. Dynamics of a real fluid and transport of contaminants in an environment with topography are governed by interaction of a

Correspondence to: Y. D. Chashechkin

(chakin@ipmnet.ru) flow with a self-induced fine structure of an initially smooth stratification. To better understand the flow past obstacles and to compare observations with analytical and numerical solutions, the typical approach has been to investigate first of all the flow at similar ranges of parameters past obstacles of simple or perfect shapes, such as strips, right circular cylinders and spheres. In this spirit, the structure of a flow around an obstacle including upstream disturbances, an internal wave field and a downstream wake have been studied both theoretically and experimentally. It is well known that vortices and vortex arrays play an important role in the transport of substances, heat and momentum. Much less is known about the effect of high gradient interfaces and reasons of their formations. Common theoretical and experimental methods are generally directed to study dynamics of regular macroscopic elements of a flow. It is difficult to investigate small-scale irregularities both in a laboratory and in the environmental conditions due to the spatial or temporal smoothing and interactions of sensors or visualization elements (seed particles, drifting balloons in the atmosphere and drifters in the ocean) with the flow. This study reports on some analytical and laboratory experiments which employ high-resolution optical techniques to provide a pattern of density gradient and velocity fields in a stratified flow around obstacles. It is important that the experimental techniques are directed to observe disturbances of the real fluid being tested and not additional substances (dye, solid particles) that change the physical properties of the medium.

\section{Analytical and numerical model}

An unbounded, incompressible, isothermal, viscous and uniformly stratified fluid is considered. An exponential distribution of the undisturbed density, $\rho_{0}(z)=\rho_{00} \exp (-z / \Lambda)$, is characterised by a constant length scale of stratification $\Lambda=|d \ln \rho / d y|^{-1}$, where $\rho_{00}=\rho_{0}(0)$ is the reference

Published by Copernicus GmbH on behalf of the European Geosciences Union and the American Geophysical Union. 
density, or by a buoyancy period, $T_{b}$, or buoyancy frequency, $N=2 \pi / T_{b}=\sqrt{g / \Lambda}$, and $g$ is the acceleration due to the gravity. To identify a set of available length scales characterising typical structural elements of a stratified flow, a system of governing equations is analysed.

The system of stratified flow governing equations in Boussinesq approximation has the form (Turner, 1973)

$$
\begin{aligned}
& \frac{\partial S}{\partial t}+\boldsymbol{u} \nabla S=\kappa_{S} \Delta S \\
& \nabla \boldsymbol{u}=0 \\
& \frac{\partial \boldsymbol{u}}{\partial t}+(\boldsymbol{u} \nabla) \boldsymbol{u}=-\frac{\nabla P}{\rho_{0}}+\nu \Delta \boldsymbol{u}+\boldsymbol{g} \beta S \\
& \rho=\rho_{0}(1+\beta S)
\end{aligned}
$$

where $P, S$ and $\boldsymbol{u}=\left(u_{x}, u_{y}, u_{z}\right)$ are pressure, salinity and velocity, respectively, $\beta$ is the salt volume expansion coefficient in the equation of state. The kinematic viscosity coefficient $v$ and salt diffusion coefficient $\kappa_{s}$ are taken as constant throughout the fluid.

A 2-D obstacle which is either an infinite horizontal strip of width $L$ or a cylinder of diameter $D$, is towed with a constant velocity $U$. In the laboratory frame of coordinates ( $\mathrm{x}, \mathrm{y}$, $\mathrm{z}$ ), the $\mathrm{z}$-axis is directed upwards, and the $\mathrm{x}$-axis is along the mean flow velocity. Boundary conditions on the body surface are no-slip for velocity, no fluxes for density and salinity and attenuation of all disturbances at infinity in the initially motionless fluid, i.e. $\left.\boldsymbol{u}\right|_{\Sigma}=0 ;\left.\quad j\right|_{\Sigma}=-\left.\kappa_{S} \nabla S\right|_{\Sigma}=0(\Sigma$ is a boundary surface).

The set of Eqs. (1) is called a singular disturbed type set if stratification is weak and viscosity is small. A complete set of solutions contains members describing large length scales components of the motion that are internal waves (Lighthill, 1978) and vortices as well as small scale ones, such as boundary layers on a solid surfaces (Schlichting, 1968; Etling, 1996). These solutions are characterised by a set of basic length scales following directly from the scaling analysis of the system (1).

The problem is characterised by the following dimensional parameters: the density $\rho_{0}(z)$ and its gradient $d \rho_{0} / d z$, kinematic viscosity $v$ and salt diffusivity $\kappa_{s}$, the velocity $U$, size of the body $D$ and gravity acceleration $g$. For methodological consistency, it makes sense to use the length scale of the flow feature or the size of the object as a unique parameter which characterises all components of the motion.

The list of intrinsic length scales of the problem includes the buoyancy scale $\Lambda=\left|d\left(\ln \rho_{0}\right) / d z\right|^{-1}$, the width $a$ of the strip or the diameter $D$ of the cylinder, the length of an attached (lee) internal wave $\lambda=U T_{b}=2 \pi U / N$, the length scale for the velocity $\delta_{u}=\nu / U$ and density $\delta_{\rho}=\kappa_{s} / U$ of the stationary boundary layers. These scales form strong inequalities $\left(\Lambda \gg D \gg \delta_{u} \gg \delta_{s} ; \Lambda \gg \lambda \gg \delta_{u}\right)$ for laboratory and environmental conditions. Additional length scales characterise diffusion-induced components of a flow structure that are $\delta_{\nu}=\sqrt{\nu / N}$ for velocity and $\delta_{s}=\sqrt{\kappa_{S} / N}$ for density (Baydulov et al., 2005). From these basic length scales, a denumerable set of derivative scales characterising secondary elements of motion $L_{c}=\sqrt[a+b+\ldots]{L_{1}^{a} \times L_{2}^{b} \times \ldots}$, for example discrete vortices and vortex arrays can be constructed. The most often used derivative scale is a viscous wave scale $L_{v}=\sqrt[3]{\Lambda \lambda \delta_{u}}=\sqrt[3]{g v} / N$, describing the geometry of vortices embedded into a 2-D density wake (Chashechkin and Voeikov, 1994). The richness of the set of length scales reflects the variety of flow structure elements and points to possible resonance effects when two scales of different nature become equal.

In this geometrical description of flow pattern, basic dimensionless parameters, namely the Reynolds number $R e=U D / \nu=D / \delta_{u}$, internal Froude number $F r=U / N D=\lambda / 2 \pi D$, Peclet number $P e=U D / \kappa_{s}=D / \delta_{\rho}$ and the ratio of scales $C=\Lambda / D$ are the ratios of the appropriate basic length scales of the problem. Equality of length scales of different nature corresponds to critical conditions of the flow. For example, widely used approximation of a "large Froude number" corresponds to $\lambda \gg D$.

The simplest case is that of uniformly moving horizontal strip with stream-wise length $L=a$ along the solid plane surface $z=0$. In this case the linearized set of governing Eqs. (1) is transformed into the standard internal wave equation for stream function $\Psi$ defining components of velocity $u_{x}=\partial \Psi / \partial z, u_{z}=-\partial \Psi / \partial x$,

$\left[\frac{\partial^{2}}{\partial t^{2}}\left(\frac{\partial^{2}}{\partial x^{2}}+\frac{\partial^{2}}{\partial z^{2}}\right)+N^{2} \frac{\partial^{2}}{\partial x^{2}}-v \frac{\partial}{\partial t}\left(\frac{\partial^{2}}{\partial x^{2}}+\frac{\partial^{2}}{\partial z^{2}}\right)^{2}\right] \Psi=0$

with no-slip boundary conditions

$$
\begin{aligned}
& \left.\frac{\partial \Psi}{\partial z}\right|_{z=0}=U \vartheta\left(x+\frac{a}{2}-U t\right) \vartheta\left(\frac{a}{2}+U t-x\right), \\
& \left.\frac{\partial \Psi}{\partial x}\right|_{z=0}=0 .
\end{aligned}
$$

where $\vartheta$ is Heaviside function, and attenuation of all disturbances at infinity. The boundary conditions must be properly changed when an inclined or vertical strip, or a cylinder, moves in a stratified fluid instead of a horizontal strip. The flow becomes more complex and has not been solved analytically up till now.

The solution of Eq. (2) is represented as the Fourier integral expansion:

$$
\begin{aligned}
& \Psi(x, z, t)=\int_{-\infty}^{\infty} e^{-i \omega t} \int_{-\infty}^{\infty} \\
& {\left[A_{w}(\omega, k) e^{i k_{w}(\omega, k) z}+B_{i}(\omega, k) e^{i k_{i}(\omega, k) z}\right] e^{i k x} d k d \omega}
\end{aligned}
$$

where roots $k_{w}$ and $k_{i}$ are solutions of the dispersion equation, corresponding Eq. (2)

$$
\omega^{2}\left(k^{2}+k_{z}^{2}\right)-N^{2} k^{2}+i \omega\left(k^{2}+k_{z}^{2}\right)^{2}=0
$$




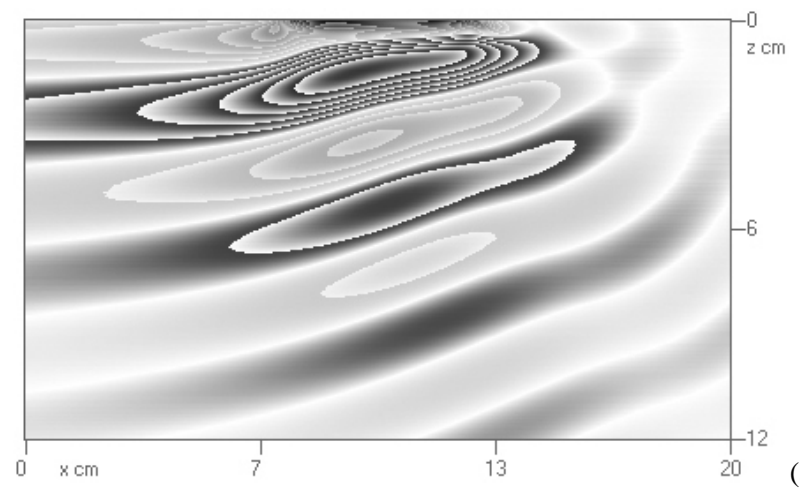

(a)

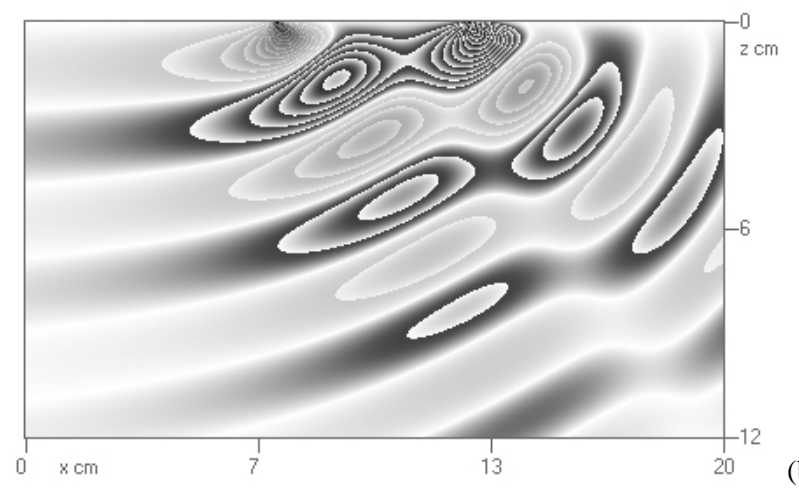

(b)

Fig. 1. Pattern of attached internal waves for horizontal (a) and vertical (b) components of velocity. Bright points on upper horizontal line indicate edges of the strip, moving from the right to the left. $T_{b}=6.28 \mathrm{~s}, a=5.5 \mathrm{~cm}, \mathrm{U}=1 \mathrm{~cm} / \mathrm{s}, \lambda=6 \mathrm{~cm}, F r=0.18, R e=550$.

Its solutions include both, roots which are regular in viscosity and correspond to waves

$k_{w}(\omega, k)=-k^{2}+\frac{i \omega}{2 v}\left[1-\sqrt{1+\frac{4 i v k^{2} N^{2}}{\omega^{3}}}\right]$

and roots singular in viscosity which correspond to boundary layers.

$k_{i}(\omega, k)=-k^{2}+\frac{i \omega}{2 v}\left[1+\sqrt{1+\frac{4 i \nu k^{2} N^{2}}{\omega^{3}}}\right]$

Substitution of Eq. (4) into the boundary conditions (3) leads to a system of algebraic equations for the spectral component of the wave amplitude

$A_{w}(\omega, k)=-A_{i}(\omega, k)=\frac{i U}{\pi k\left(k_{q}-k_{i}\right)} \sin \frac{k a}{2} \delta(\omega-k U)$

Substitution of the solution (6) into (4) and integration give the resultant expression for the stream function

$$
\begin{aligned}
\Psi(x, z, t)= & \frac{i U}{\pi} \int_{-\infty}^{\infty} \frac{1}{k} \sin \frac{k a}{2} e^{i k(x-U t)} \\
& \frac{e^{i k_{w}(k U, k) z}-e^{i k_{i}(k U, k) z}}{k_{w}(k U, k)-k_{i}(k U, k)} d k
\end{aligned}
$$

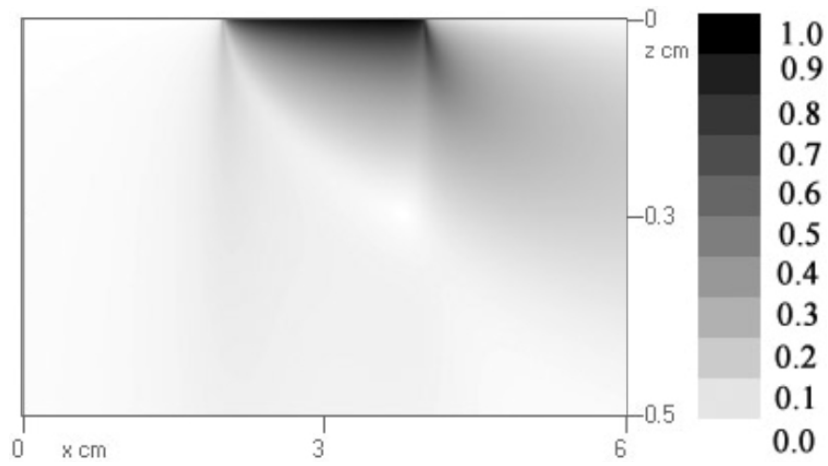

Fig. 2. Module of field velocity within the boundary layer on the $\operatorname{strip}\left(T_{b}=14 \mathrm{~s}, \mathrm{a}=2 \mathrm{~cm}, \mathrm{U}=1 \mathrm{~cm} / \mathrm{s}, \lambda=14 \mathrm{~cm}, F r=1.12, R e=200\right)$.

From Eq. (7), it follows that the field of lee waves is transient ahead and stationary behind the source in the reference frame. Detailed analytical and numerical analysis of Eq. (7) was presented by Chashechkin and Bardakov (2004).

A visualization of the exact solution (7) for the vertical component of velocity and vorticity by the modified method of isopleths in Fig. 1 makes it possible to reveal not only the complete structure of transient leading and stationary attached internal waves, but also details of the fine structure of the boundary layer. Wave perturbations near the plate are more pronounced for the horizontal velocity than for the vertical one. Moreover, the number of perturbation peaks in a single wave field turns out to be different for different wave components (one peak band for the horizontal component and two bands for the vertical one).

The transient upstream waves are non-stationary. The phase surface slope to the horizon characterises local frequency values.

The detailed structure of the module of field velocity within the boundary layer is shown as a magnified continuous-tone image in Fig. 2. Both leading and trailing edges of the plate incorporate singular perturbations with the vertical velocity oriented at first toward the fluid and, then, toward the plate. The edge singularities of the horizontal velocity are much less pronounced. The thickness of the Prandtl boundary layer (with a typical scale of $\delta_{u}=v / U$ ) monotonously increases with distance from the leading edge the same way as in laminar flow of a homogeneous liquid (Schlichting, 1968). The boundary layer is detached from the trailing edge into the liquid. The contrast of singular components of the boundary layer varies with changes in the flow parameters. The complicated structure of boundary layers at a horizontally moving plate indicates that it is impossible to model the formation of attached internal waves near a real obstacle in terms of a set of singular mass or force sources.

The complex structure of a calculated flow pattern indicates that sensitive instruments must be used in experiments for observation of flow field, which can visualize simultaneously regular large-scale and singular small-scale 

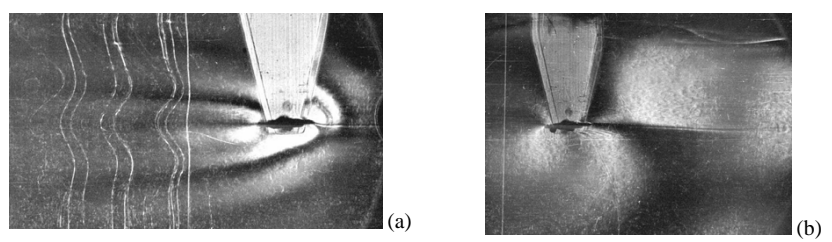

Fig. 3. Pattern of flow around the horizontal strip moving from left to right $\left(a=2.5 \mathrm{~cm}, \mathrm{~T}_{b}=14.0 \mathrm{~s}, \mathrm{C}=5000\right)$; (a) $\mathrm{U}=0.1 \mathrm{~cm} / \mathrm{s}$, $\mathrm{Re}=25, \mathrm{Fr}=0.09, \lambda=1.4 \mathrm{~cm}, \delta_{u}=0.1 \mathrm{~cm}, \alpha=0^{\circ}$, "slit-thread"; (b) $\mathrm{U}=0.9 \mathrm{~cm} / \mathrm{s}, \operatorname{Re}=225, \mathrm{Fr}=0.8, \lambda=12.6 \mathrm{~cm}, \delta_{u}=0.011 \mathrm{~cm}, \alpha=3^{\circ}$, "slit-knife".

components of flow. Only sensitive Schlieren instrument and marker lines satisfy this condition.

\subsection{Experimental set-up and techniques}

The experiments were conducted in a rectangular transparent tank $\left(220 \times 40 \times 60 \mathrm{~cm}^{3}\right)$ with optical windows, filled with linearly stratified brine $\left(\nu=0.01 \mathrm{~cm}^{2} / \mathrm{s}, \kappa_{s}=1.43 \cdot 10^{-5} \mathrm{~cm}^{2} / \mathrm{s}\right)$. A side view of a flow is observed by a Russian Schlieren instrument IAB458 with a field of view of $23 \mathrm{~cm}$ in diameter. Various types of light-cutting diaphragms were used, namely a flat knife, producing conventional Schlieren pattern; Maksoutov's thread, as well as a horizontal grating producing natural "rainbow" colour Schlieren image (Chashechkin, 1999) was used. The Schlieren instrument has a spatial resolution better than $0.1 \mathrm{~mm}$. Colour images are reproduced here in a grey scale.

A horizontal cylinder or a plate placed vertically, horizontally or under some angle to the horizontal, $\alpha$, is towed by a carriage. The experimental conditions presented in Table 1 correspond to laminar, transient and turbulent flow regimes following the classification by Boyer et al. (1989).

\subsection{Results}

\subsection{Horizontal and inclined strips}

Black-and-white Schlieren images of the flow around the horizontal strip, towed from the right to the left, in the weak density gradient fluid are shown in Fig. 3. The sloping straight rays ahead of the obstacle (to its left) visualize upstream transient internal waves. Crests and troughs of the waves are matched with the appropriate circular phase surfaces of attached (lee) internal waves past the strip. The wave pattern corresponds to that calculated from expression (7). The twin grey lines past the strip (Fig. 3a) show the troughs of the internal waves while dark lines mark the crests. Thin high gradient interfaces in the downstream wake originate from the rear edge of the strip.

Distorted vertical lines ahead the obstacle in Fig. 3 are density markers visualising vertical profiles of the velocity.

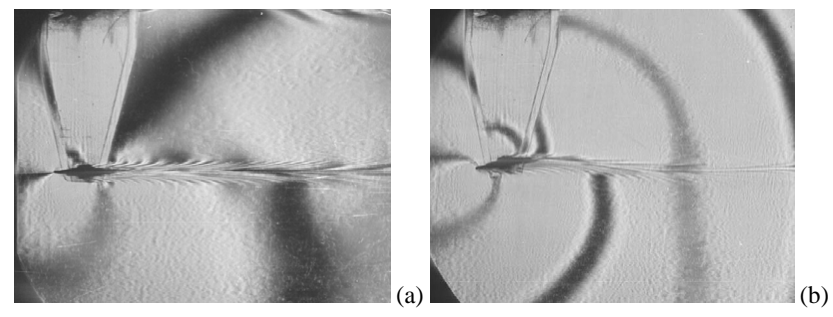

Fig. 4. Pattern of flow around strip moving from right to left produced by "slit-thread" method, $T_{b}=7.5 \mathrm{~s}, a=2.5 \mathrm{~cm}, \mathrm{C}=560$; (a) $U=2.3 \mathrm{~cm} / \mathrm{s}, \mathrm{Re}=575, \mathrm{Fr}=1, \lambda=17.3, \delta_{u}=0.004, \alpha=0^{\circ}$; (b) $U=1.4 \mathrm{~cm} / \mathrm{s}, \operatorname{Re}=350, \mathrm{Fr}=0.67, \lambda=10.5, \delta_{u}=0.1 \mathrm{~cm}, \alpha=12.5^{\circ}$.

The height of the central blocked liquid area is larger than the strip thickness. The vertical thread in the right part of the image in Fig. $3 b$ is the reference line to measure the markers displacements. Contours of the markers in the upper part of the Fig. 3a indicate an upstream influence of both the obstacle and the vertical blades holding the obstacle.

With the towing velocity increased, the intensity of the upstream disturbances decreased, the length of internal waves increased and a new structural component of the flow was observed. Conventional Schlieren method reveals four thin short sloping interfaces in the downwind side below the sloping strip terminating with the free sharp edges (Fig. 3b). Their leading edges closely attach to the horizontal boundary layer on the strip. The thickness of the interfaces is less than $0.1 \mathrm{~cm}$, and the distance between them is about $0.5 \mathrm{~cm}$. The slope of the interfaces gradually increases with the distance from the plate. The lift force distorts the thin density wake and displaces crests and troughs (Chashechkin and Mitkin, 2001). The density wake position is restored due to buoyancy forces and, with distance, it gradually comes back to the body path.

With the buoyancy frequency increased, the flow pattern becomes more complicated and a total number of transverse streaks past the strip increases (Fig. 4). Near the obstacle, their leading edges are directed horizontally, while their sharp outer edges are oriented almost vertically. The streaky wake remains rather narrow due to the buoyancy forces suppressing the vertical displacement of fluid particles. With time and with distance from the strip, the slopes (with respect to the horizontal) of the individual streaks and the height of the whole structure decrease. Due to the shear flow inside the velocity wake, the streaks elongate in the horizontal direction. They are gradually smoothed by molecular diffusion and disappear.

The lift force changes the flow pattern around the sloping strip. If the first trough and first crests in Fig. $4 \mathrm{~b}$ are located at the strip's leading edge in the upper semi-space and its rear in the lower semi-space, respectively, the following troughs in the both semi-spaces contact each other through the thin density wake. The central interface of the wake becomes 
Table 1. Conditions of experiments.

\begin{tabular}{ccccccc}
\hline obstacle & diameter/width, cm & $T_{b}, \mathrm{~s}$ & $\mathrm{U}, \mathrm{cm} / \mathrm{s}$ & $\mathrm{Re}$ & $\mathrm{Fr}$ & $\mathrm{C}$ \\
\hline cylinder & $1.5 ; 2.5 ; 5.0 ; 7.6$ & $5.3-30$ & $0.01-6$ & $1.5-2100$ & $0.004-8.5$ & $100-15000$ \\
plate & 2.5 & $5.3-30$ & $0.01-6$ & $2.5-1500$ & $0.003-11$ & $300-9000$ \\
\hline
\end{tabular}
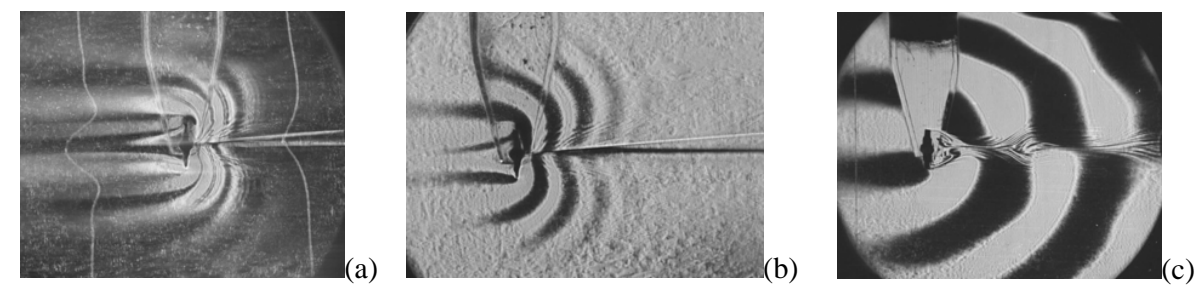

Fig. 5. Pattern of flow around a vertical strip $\left(H_{c}=2.5 \mathrm{~cm}\right)$ moving from right to left; $(\mathbf{a}, \mathbf{b})-T_{b}=12.5 \mathrm{~s}, U=0.1 \mathrm{~cm} / \mathrm{s}, \mathrm{C}=1600, \mathrm{Re}=25$, $\mathrm{Fr}=0.08, \lambda=1.25, \delta_{u}=0.1$, "slit-thread" and "slit-knife" methods; $(\mathbf{c})-T_{b}=17.4 \mathrm{~s}, U=0.3 \mathrm{~cm} / \mathrm{s}, \mathrm{C}=3000, \operatorname{Re}=75, \mathrm{Fr}=0.33, \lambda=5.22, \delta_{u}=0.033$, "slit-knife" method.

wavy-shaped with a spatial period equal to the length of the attached internal waves $\lambda=U \cdot T_{b}$. In the upper semi-space, the streaks in the upwind side of the obstacle are arranged with relatively large intervals. They separate only from the rear edge of the strip.

As a contrast, in the downwind strip side, the streaky structure separates along the entire surface, starting at the leading edge of the strip. Their sharp trailing ends form large angles with the obstacle trajectory. The overall length of the domain occupied by the streaky structures and the thickness of individual interfaces are the same, but the total numbers of sloping interfaces are different in the upper and lower hemispaces, respectively.

The thickness of the boundary layer is small in the upwind side of the obstacle and large in the downstream side where the interfaces have merged into a uniform disturbance. Due to the baroclinicity, this domain is characterized by a high level of vorticity in the form of superposed interfaces. Differences in the thickness and colour of curved strips, illustrating crests and troughs of internal waves in Figs. 4a, b, are caused by differences in wave amplitudes, mutual locations and thickness of the illuminating slits and cutting threads.

\subsection{Vertical strips and cylinders}

To illustrate the effect of the strip position on flow structure, two first images shown in Fig. 5 present the same flow pattern, visualized by the two different Schlieren methods. The basic flow elements, namely internal waves and the downstream wake with fine internal structure, are reproduced oneto-one in independent experiments. An increase of the crest and troughs lengths in Fig. 5a, comparably to Fig. 5b, is due to a higher sensitivity of slit-thread Schlieren method under the given conditions of the instrument tuning. The curved dark and light strips visualising attached internal wave past the body are imperfect circular arcs, where their distortion is caused by the Doppler effect inside the velocity wake shear flow.

The vertical markers indicate a profile of the velocity horizontal component. An upstream central jet representing blocked fluid is more pronounced than the downstream wake. The height of the velocity wake exceeds the density wake thickness bounded by two sloping interfaces. The density wake wedge contacts the vertical strip through the single central interface whose length is about $2 \mathrm{~cm}$. Due to buoyancy forces, the high gradient boundary layers separating from upper and lower edges of the strip converge to the central horizontal plane. At a given flow regime, thin elongated interfaces occupy the whole velocity wake. The curves of attached internal waves crests and troughs penetrate through the interfaces with little distortions. The sharp edges of the strip generate their own set of upstream disturbances.

With the velocity increased, the vorticity is accumulated in the rear part of the strip in Fig. 5c. Because shedding from the rear vortices pair is suppressed by attached internal waves, the wake past the obstacle is thin but then expands. Further the wake expands following the phase structure of the internal waves. The fine scale structure inside the wake is always observed as long as the attached internal waves exist. The flow pattern becomes more complex past a large bluff body, replacing some volume of fluid.

The main difference is the direct formation of interfaces, which really represent vortex sheets in the stratified fluid interior. Traditionally it is supposed that vorticity is diffused inside the fluid from highly sheared flows on solid boundaries. Direct formation of singular interfaces inside the attached wave, which have no features on their leading and trailing edges, demonstrate that elements with high vorticity 

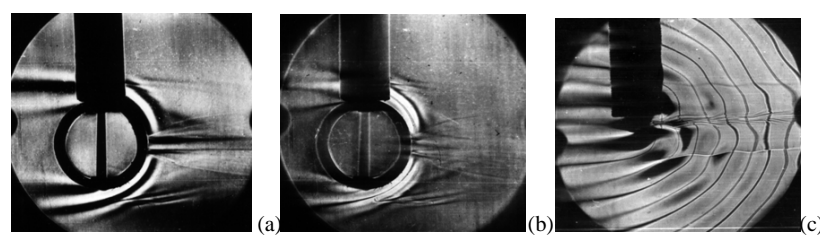

Fig. 6. Conventional (a) and Maksoutov's (b, c) Schlieren images of the flow past a cylinder with soaring interfaces; (a), (b) $-\mathrm{D}=7.6 \mathrm{~cm}, \mathrm{~T}_{b}=20.5 \mathrm{~s}, \mathrm{C}=1400, \mathrm{U}=0.04 \mathrm{~cm} / \mathrm{s}, \mathrm{Re}=30, \mathrm{Fr}=0.017$, $\lambda=0.82, \delta_{u}=0.25 ;(\mathrm{c})-\mathrm{D}=1.5 \mathrm{~cm}, \mathrm{~T}_{b}=6 \mathrm{~s}, \mathrm{C}=600, \mathrm{U}=0.52 \mathrm{~cm} / \mathrm{s}$, $\operatorname{Re}=78, \operatorname{Fr}=0.33, \lambda=3.12, \delta_{u}=0.02$.

can be formed inside the flow field. Examples of this flow type are presented in Fig. 6.

The geometry of the downstream density wake, to the right of the cylinder in Fig. 6, is defined by two almost horizontal interfaces and two sloping interfaces, which contact in the line of a boundary layers operation on the cylinder surface. These interfaces separate three different water masses inside the density wake.

The most interesting elements in the pattern of flow in Fig. 6 are two systems of soaring discontinuities that do not touch the body surface. Their leading and trailing edges do not contact with any singular surface in the flow. They are placed inside the smooth attached internal wave field and formed due to interaction of waves and the shear flow past the body. The interfaces are unchanged when the method of visualization is changed (Figs. 6a, b). Molecular diffusion smoothes the gradients over the interface, which causes the trailing edge of the structure to be less defined than the leading edge. The thickness of the individual components of the soaring interfaces does not exceed $1.5 \mathrm{~mm}$, which is markedly less than the length of the attached internal wave $(\lambda=1.1 \mathrm{~cm})$.

With an intensification of the initial density gradient the wave motion as well as the contrast and length of the soaring interfaces increases (Fig. 6c). Internal waves also disturb the interfaces in to wavy surfaces. In this range of parameters, the density wake consists of a sequence of "vortex bubbles" which are bounded by multilayered envelopes (Boyer et al., 1989). The distance between "bubbles" correlates with the attached internal wavelength $\left(\lambda=U T_{b}\right)$. The shape of wave crests is distorted by the shear flows inside the velocity wake. Because of this crests and troughs above and below the body central plane in Fig. 6c look like the ones reconnected through the density wake. That is in contrast with all previous photos where the wave field was also antisymmetric. In the outer area the downstream wave field is antisymmetric.

The interaction of attached internal waves with soaring interfaces leads to formation of leading edge vortices and vortex systems (Chashechkin and Mitkin, 2005). The temporal evolution of this flow is presented in Fig. 7. The complex
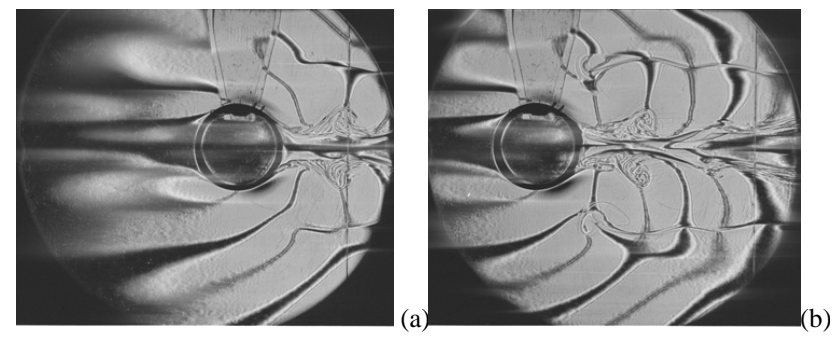

Fig. 7. Evolution of the flow pattern around horizontal cylinder: subsequent formation of soaring interfaces and vortex pairs on leading edges of these interfaces above and below cylinder $\left(T_{b}=13 \mathrm{~s}, D=5 \mathrm{~cm}, \mathrm{C}=850, U=0.35 \mathrm{~cm} / \mathrm{s} ; F r=0.14, \quad R e=165\right.$, $\lambda=4.55, \delta_{u}=0.03$ ): (a) - soaring interfaces, $\tau=t / T_{b}=3.9$, (b) - singular vortex pairs on the leading edges of the soaring interfaces, $\tau=5.8$.

downstream wake originates from the rear vortex, which is directly adjacent to the body. Under internal wave action the height of the wake as a whole rapidly decreases but a pair of immersed vortices, which are stationary with respect to the obstacle, increases the wake size. Downstream of the vortices, the vertical wake size decreases again and attains a minimum. The position of vortex pairs immersed in the wake is strongly synchronized with the phase structure of attached waves. Lines of crests (solid dark lines) and of depressions (double grey lines) advance precisely to the centre of the vortices immersed in the wake.

The pattern of attached internal waves preserves its regularity until the density-gradient field loses its uniformity. Attached internal waves incident on interfaces are partly reflected and partly transformed into internal boundary flows that, in turn, amplify arising stratification inhomogeneities.

Gradually, the stratification gradients become more and more pronounced and begin to be recorded by the shadow instrument. Novel structure components appear in the flow pattern, namely, solitary isolated interfaces (Fig. 7a). The pointed leading edge of the soaring interface is located in the area of maximum amplitudes of attached internal waves.

The analysis of changes in the location and shape of the crests and depressions of attached internal waves allows us to reveal the mechanism of the vortex-system formation at the leading edge of the interface. This mechanism is closely related to the arising spatial homogeneity of the soaring interface structure.

A moving large body changes the initial gradient profile and creates strong down stream shear flow. Propagating attached internal waves non-uniformly change their slope, shapes of crests and troughs and wavelengths due to the Doppler effect and a local relative frequency value changes. Internal waves crossing the soaring interface produce additional boundary currents on its sides. The value and direction of these currents depends on the wave phase. As one can see in Fig. 7b the first grey line outgoing from the bubble vortex 
below the density wake meets on the interface with dark line illustrating the crest of attached internal waves. It means that in some domains on different sides of the interfaces crests and troughs of the attached downstream waves are placed. Their meet each other at the same point on the soaring interfaces (in Fig. 7 the crest centre corresponds to the solid dark line). Thus, in the head segment above and below the soaring the interface, wave fields are present for which the wavy flows have oppositely directed vertical components. These components are transformed into spreading horizontal flows on the soaring interfaces. This complicated flow structure is stabilized by high-density gradients at the interface. All arising inhomogeneities of the density distribution are of a purely deformation nature and appear as a result of smooth liquid-particle transfer from different horizons.

So near the leading edge, the soaring interface is considered as an analog of a shock wave, because in its vicinity the continuity condition for the velocity normal component is violated. In high-velocity hydrodynamics, shock waves separating spatial domains with different values of the velocity normal component have been thoroughly studied. With increasing velocity, the size of leading edge vortex pair is enlarged and a new vortex system is formed in the vicinity of the first depression of the density wake.

\section{Discussion}

These observations demonstrate the existence of transient streaky structures past the uniformly moving strip and singular interfaces in stratified flows past the cylinder. Formation of streaky structures is caused by action of singularities of leading and trailing edges due to finite size of the obstacle. The edges singularities exist in the linear model of the flow. Strong interaction of all regular and singular components of the flow leads to multiplying of streaks oriented along streamlines.

Formation of soaring vortices in the wave wake past the horizontal cylinder is caused by interaction of internal waves and interfaces arising in the initially smooth density and velocity fields. Appearance of interfaces strongly affects transport of contaminants on several reasons. Firstly the flow in opposite directions on the different sides of the interfaces brings contaminants in to the high gradient region, where then a new thin flow develops which transports the contaminants. Spatially correlated slow transport by incoming flows can produce rather strong narrow currents providing a redistribution of mass or concentration and results in fast transport over large distances. The accumulations of contaminants on interfaces, which form the outer envelope of the density wake and soaring interfaces was observed in laboratory tanks too.

\section{Conclusion}

Theoretical and experimental studies of flows around obstacles in a continuously stratified fluid were performed. The flow was found to contain both, regular components in the form of internal waves and vortices, and singular components in the form of boundary layers and internal "boundary" currents. The internal boundary currents manifested themselves as interfaces in the stratified fluid. The phase pattern of the observed attached internal waves agreed with those calculated from the theory derived from the governing equations without any additional parameters. That means that calculation of downstream internal waves and positions of soaring interfaces needs detailed knowledge of density gradient and velocity profiles with details of their fine structure.

Transient streaky structures were for the first time observed in the downstream laminar wake of a horizontally moving strip. These structures can be predecessors of wellknown vortex loops and hairpin vortices observed in more high-speed boundary layers (Kozlov at al., 1998).

Evolution of soaring interfaces, which are low velocity analogues of shock waves, and the formation of singular vortex pairs on their leading edges were investigated.

The set of all regular and singular components of flow form a unified system. All components of the system form and disappear synchronously in spite of their difference of the characteristic scales. These strong interactions between singular and regular components may explain the fast evolution of environmental systems in certain critical conditions. New sensitive methods and instruments must be developed for observations and measurements of these phenomena in the laboratory and in the environment.

Acknowledgements. The research is supported by the Russian Academy of Sciences (Program OE-14 "Dynamics and acoustics of non-homogeneous fluids, gas-liquid mixtures and suspensions") and the Russian Foundation for Basic Research (Grant 05-0564090).

Edited by: R. Grimshaw

Reviewed by: W.-G. Früh and another referee

\section{References}

Baydulov, V. G., Matyushin, P. V., and Chashechkin, Y. D.: Structure of diffusion induced flow near a sphere in a continuously stratified fluid, Doklady Physics, 50(4), 195-199, 2005.

Boyer, D. L., Davies, P. A., Fernando, H. J. S., and Zhang, X.: Linearly stratified flow past a horizontal circular cylinder, Phil. Trans. R. Soc. London, A328, 501-528, 1989.

Chashechkin, Y. D. and Mitkin, V. V.: Formation of Vortices on Soaring Interfaces, in: Stratified Flows Behind a Cylinder, Doklady Physics, 50(9), 452-456, 2005.

Chashechkin, Y. D. and Voeikov, I. V.: Vortex systems past a cylinder in a continuously stratified fluid, Izvestiya - Atmos. Ocean. Phys., 29(6), 787-795, 1994. 
Chashechkin, Y. D. and Bardakov, R. N.: Two-Dimensional Attached Internal Waves and Concomitant Boundary Layers, Yu. D. Doklady Earth Sciences, 397(5), 677-681, 2004.

Chashechkin, Y. D. and Mitkin, V. V.: An effect of a lift force on the structure of attached internal waves in a continuously stratified fluid, Doklady Physics, 46-6, 425-428, 2001.

Etling, D.: Theoretische Meteorologie, Verlag Vieweg, Braunschweig/Wiesbaden, 318 p., 1996.

Kozlov, V. V., Westin, K. J. A., Bakchinov, A. A., and Alfredsson, P. H.: Experiments on localized disturbances in a flat plate boundary layer. Part 1 . The receptivity and evolution of a localized free stream disturbances, Eur. J. Mech. Fluids, 17, 823-846, 1998.
Lighthill, J.: Waves in fluids, Cambridge university press, Cambridge, 1978.

Schlichting, H.: Boundary Layer Theory, 6th ed., McGraw-Hill, New York, 1968.

Turner, J. S.: Buoyancy Effects in Fluids, Cambridge Univ. Press, Cambridge, UK, 368 pp, 1973. 